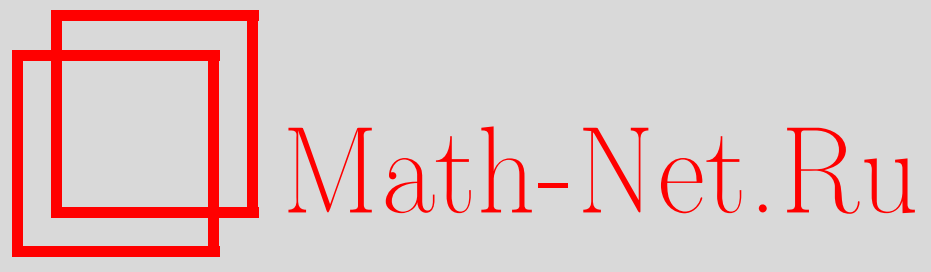

Н. В. Бударина, Ф. Гетце, Расстояние между сопряженными алгебраическими числами в кластерах, Mатем. заметки, 2013, том 94, выпуск 5, 780-783

DOI: https://doi.org/10.4213/mzm10334

Использование Общероссийского математического портала Math-Net.Ru подразумевает, что вы прочитали и согласны с пользовательским соглашением http://www . mathnet.ru/rus/agreement

Параметры загрузки:

IP : 52.87 .193 .239

26 апреля 2023 г., 15:54:17

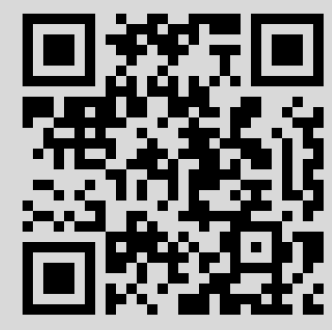




\section{Расстояние между сопряженными алгебраическими числами в кластерах}

\section{Н. В. Бударина, Ф. Гетце}

Для натуральных чисел $n \geqslant 2$ и $Q>1$ введем класс целочисленных многочленов $P(x)=$ $a_{n} x^{n}+a_{n-1} x^{n-1}+\cdots+a_{1} x+a_{0}$ :

$$
\mathcal{P}_{n}(Q)=\{P \in \mathbb{Z}[x]: \operatorname{deg} P=n, H(P) \leqslant Q\},
$$

где $H=H(P)=\max _{0 \leqslant j \leqslant n}\left|a_{j}\right|$ - высота $P$. Всюду далее, $\alpha_{1}, \ldots, \alpha_{n} \in \mathbb{C}, \alpha_{i} \neq \alpha_{j},-$ корни $P$, а $c_{1}=c_{1}(n), c_{2}=c_{2}(n), \ldots$ - величины, зависящие от $n$ и не зависящие от $H$ и $Q$. Далее $a \ll b$ означает, что существует константа $c>0$ такая, что $a \leqslant c b$; выражение $a \asymp b$ эквивалентно $a \ll b \ll a$. Существует много характеристик $P$, связанных с его корнями. Например, производная $P^{\prime}$ в корне $\alpha_{1}$ :

$$
P^{\prime}\left(\alpha_{1}\right)=a_{n}\left(\alpha_{1}-\alpha_{2}\right)\left(\alpha_{1}-\alpha_{3}\right) \ldots\left(\alpha_{1}-\alpha_{n}\right),
$$

дискриминант $D(P)$ :

$$
D(P)=a_{n}^{2 n-2} \prod_{1 \leqslant i<j \leqslant n}\left(\alpha_{i}-\alpha_{j}\right)^{2} .
$$

Оценки $\left|P^{\prime}\left(\alpha_{1}\right)\right|$ и $|D(P)|$ как сверху, так и снизу имеют важное значение в теории трансцендентных чисел и теории диофантовых приближений [1]. На этих оценках основано доказательство проблемы Малера [2], аналога метрической теоремы А.Я. Хинчина для многочленов произвольной степени, получение точного значения размерности Хаусдорфа множеств действительных и комплексных чисел с заданной мерой трансцендентности [1].

В последние годы началось систематическое изучение величин $\left|\alpha_{i}-\alpha_{j}\right|$ для различных сопряженных алгебраических чисел $\alpha_{i}$ и $\alpha_{j}$, и более общей задачи о кластерах $M_{k}=$ $\prod_{1 \leqslant i<j \leqslant k}\left|\alpha_{i}-\alpha_{j}\right|$ различных корней $\alpha_{1}, \ldots, \alpha_{k}$ целочисленного многочлена $P$ степени $n$, где $2 \leqslant k \leqslant n$. Оценка снизу для $\left|\alpha_{i}-\alpha_{j}\right|$ была получена Малером [3]:

$$
\left|\alpha_{i}-\alpha_{j}\right|>\sqrt{3}(n+1)^{-(2 n+1) / 2} H(P)^{-n+1},
$$

которая является частным случаем оценки снизу

$$
\prod_{1 \leqslant i<j \leqslant k}\left|\alpha_{i}-\alpha_{j}\right| \gg H(P)^{-n+1}
$$

справедливой для любого целочисленного многочлена $P$ степени $n$ и имеющего, по крайней мере, $k \geqslant 2$ различных корней $\alpha_{1}, \ldots, \alpha_{k}$.

Пусть $n, k \in \mathbb{N}$ удовлетворяют условию $2 \leqslant k \leqslant n$. Обозначим через $E(n, k)$, соответственно $E_{\mathrm{irr}}(n, k)$, инфимум действительных чисел $\delta$, для которых неравенство

$$
\prod_{1 \leqslant i<j \leqslant k}\left|\alpha_{i}-\alpha_{j}\right| \geqslant H(P)^{-\delta}
$$

выполняется для каждого целочисленного многочлена $P$, соответственно целочисленного неприводимого многочлена $P$, степени $n$ и достаточно большой высоты с различными корнями $\alpha_{1}, \ldots, \alpha_{k}$. Из (1) следует, что $E_{\mathrm{irr}}(n, k) \leqslant E(n, k) \leqslant n-1$ для любых $n$ и $k$, удовлетворяющих $2 \leqslant k \leqslant n$.

Работа выполнена при поддержке гранта CRC 701 университета Билефельда.

DOI: $10.4213 / \mathrm{mzm} 10334$ 
Существование близких сопряженных чисел доказано в работах [4]-[8]. K настоящему времени известны только следующие точные оценки

$$
E_{\text {irr }}(2,2)=E(2,2)=1, \quad E_{\text {irr }}(3,2)=E(3,2)=2
$$

(см. [6]). Наилучшие к настоящему времени оценки снизу

$$
\begin{aligned}
& E_{\mathrm{irr}}(n, 2) \geqslant \frac{n}{2}+\frac{n-2}{4(n-1)} \quad \text { для } \quad n \geqslant 4 \text {, } \\
& E(n, 2) \geqslant \frac{2 n-1}{3} \quad \text { для } n \geqslant 4 \text {, }
\end{aligned}
$$

доказаны в [7] и [8] соответственно.

Относительно нижних оценок для $E(n, k)$ и $E_{\operatorname{irr}}(n, k), k>2$, в [6] доказано следующее:

$$
\begin{array}{rlrl}
E_{\mathrm{irr}}(n, n-1) & =n-1 & & \text { для } n \geqslant 4, \\
E(n, k) & \geqslant \frac{k-1}{k} n, \quad E_{\mathrm{irr}}(n, k) \geqslant \frac{k-1}{k} n \quad \text { для } k \geqslant 3, \quad n=t k, \quad t \in \mathbb{N} .
\end{array}
$$

В работе [8] показано, что для любого целого $k \geqslant 3$ существует рациональное число $c_{1}(k)$ такое, что при любом $n \geqslant k$ справедливо неравенство

$$
E(n, k) \geqslant \frac{k}{k+1} n-c_{1}(k)
$$

В данной работе мы получаем в большей части диапазона изменения $k$ лучшие, чем в $(2)$, оценки для $E_{\mathrm{irr}}(n, k)$. Более того, мы даем оценки снизу для количества многочленов $P \in \mathcal{P}_{n}(Q)$, для которых такие оценки выполняются.

Teоpema 1 . Пусть $2 \leqslant k \leqslant n u 0 \leqslant \gamma \leqslant 2(n+1) /(k(k+1))$. Тогда существует $N_{1}$, где $N_{1}>c_{2} Q^{n+1-((k-1)(k+2) / 2) \gamma}$, многочленов $P \in \mathcal{P}_{n}(Q)$ mаких, что

$$
E_{\text {irr }}(n, k) \geqslant \frac{k(k-1)}{2} \gamma .
$$

СлЕДСтвие 1. При $\gamma=2(n+1) /(k(k+1))$ получаем $E_{\mathrm{irr}}(n, k) \geqslant((k-1) /(k+1))(n+1)$, что при $k>c_{3} n$, где $c_{3}<1$, улучшает оченку (2).

Если $k=n$, то $E_{\mathrm{irr}}(n, n) \geqslant n-1$ и $N_{1}>c_{4} Q^{2 / n}$, т.е. в этом случае оченки сверху и снизу для кластера $M_{n}$ совпадают.

ЗАмечАние 1. Интересным является тот факт, что оценки для кластера

$$
M_{k}^{\prime}=\left|\alpha_{1}-\alpha_{2}\right|\left|\alpha_{1}-\alpha_{3}\right| \cdots\left|\alpha_{1}-\alpha_{k}\right|, \quad 2 \leqslant k \leqslant n,
$$

который является нормированным значением модуля производной в корне (или его частью), получаются, а может и являются, такими же как и для одного сомножителя $\left|\alpha_{1}-\alpha_{i}\right|$. Из теоремы 1 можно получить, что существует не менее $c_{5} Q^{(n+1) / 3}$ многочленов $P \in \mathcal{P}_{n}(Q)$, для различных сопряженных корней которых выполняются неравенства

$$
\left|\alpha_{1}-\alpha_{2}\right| \ll Q^{-(n+1) / 3}, \quad M_{k}^{\prime} \ll Q^{-(n+1) / 3}, \quad k>2 .
$$

ДоказАтельство теОРемы 1 . Обозначим через $B_{1}$ множество точек $x$ из интервала $I \subset[-1 / 2,1 / 2]$, для которых при

$$
v_{0} \geqslant v_{1} \geqslant \cdots \geqslant v_{m} \geqslant-1, \quad 0 \leqslant j \leqslant m, \quad \sum_{i=0}^{m} v_{i}=n-m
$$


система неравенств

$$
\begin{aligned}
\delta_{0} Q^{-v_{j}} & <\left|P^{(j)}(x)\right|<c_{0} Q^{-v_{j}}, & 0 & \leqslant j \leqslant m, \\
\delta_{0} Q & <\left|P^{(j)}(x)\right|<c_{0} Q, & m+1 & \leqslant j \leqslant n
\end{aligned}
$$

имеет решение в неприводимых многочленах $P \in \mathcal{P}_{n}(Q)$. Согласно определению $E_{\mathrm{irr}}(n$, $m+1)$ имеем $2 \leqslant m+1 \leqslant n$.

Для приложений системы неравенств (4) потребуем, чтобы при $1 \leqslant j \leqslant m$ последовательность

$$
d_{j}=v_{j-1}-v_{j}, \quad d_{m+1}=v_{m}+1
$$

не возрастала. Упорядочим корни многочлена $P$ относительно точки $x$ так, чтобы

$$
\left|x-\alpha_{1}\right| \leqslant\left|x-\alpha_{2}\right| \leqslant \cdots \leqslant\left|x-\alpha_{n}\right| .
$$

В работе [4] доказана следующая лемма, которая имеет много приложений при оценках дискриминантов и результантов.

Лемма 1. Пусть данъ иелье числа $n \geqslant 2$ и $m \in[0, n-1]$. Тогда существуют положительные постоянные $\delta_{0}=\delta_{0}(n)$ u $c_{0}=c_{0}(n)$ такие, что $\mu B_{1}>(3 / 4) \mu I$.

Лемма 2. Пусть $x \in B_{1}$. Тогда при выполнении условия (5) корни $\alpha_{1}, \alpha_{2}, \ldots, \alpha_{m+1}$ многочлена $P$ удовлетворяют неравенствам

$$
\left|x-\alpha_{j}\right| \ll Q^{-v_{j-1}+v_{j}}, \quad 1 \leqslant j \leqslant m, \quad\left|x-\alpha_{m+1}\right| \ll Q^{-v_{m}-1} .
$$

Лемма 2 доказана в [9].

Возьмем $\gamma=1+v_{m}, \gamma=v_{j-1}-v_{j}, 2 \leqslant j \leqslant m$ и $v_{0}-v_{1} \geqslant \gamma$. Тогда

$$
v_{m}=-1+\gamma, \quad v_{m-1}=v_{m}+\gamma=-1+2 \gamma, \quad \ldots, \quad v_{1}=v_{2}+\gamma=-1+m \gamma
$$

Из равенства $\sum_{i=0}^{m} v_{i}=n-m$ получаем $v_{0}=n-(m(m+1) / 2) \gamma$. Вычислим $v_{0}-v_{1}=$ $n+1-(m(m+3) / 2) \gamma$. Поскольку $v_{0}-v_{1} \geqslant \gamma$, то $0 \leqslant \gamma \leqslant 2(n+1) /((m+1)(m+2))$, откуда следует, что

$$
\begin{aligned}
\prod_{1 \leqslant i<j \leqslant m+1}\left|\alpha_{i}-\alpha_{j}\right| & \leqslant \prod_{1 \leqslant i<j \leqslant m+1}\left(\left|x-\alpha_{i}\right|+\left|x-\alpha_{j}\right|\right) \\
& \ll \prod_{1 \leqslant i<j \leqslant m+1}\left|x-\alpha_{j}\right| \asymp Q^{-(m(m+1) / 2) \gamma} .
\end{aligned}
$$

Таким образом, в точке $x \in B_{1}$ мы построили многочлен $P$ такой, что для кластера $M_{m+1}$ справедливо неравенство (6) и $\left|x-\alpha_{1}\right|<c_{6} Q^{-v_{0}+v_{1}}$. Этот же многочлен $P$ может быть решением (4)-(6) и для других точек $x \in B_{1}$, в окрестности которых есть другие корни многочлена $P$. Мера объединения таких интервалов, взятая по $n$ корням многочлена $P$ не превосходит $2 n c_{6} Q^{-v_{0}+v_{1}}$. Возьмем другую точку $x^{\prime} \in B_{1}$ вне этого объединения и построим новый многочлен с кластером корней $M_{m+1}$, удовлетворяющим (6) и т.д. Поскольку по лемме 1 множество $B_{1}$ имеет меру, большую $(3 / 4) \mu I$, то в результате построим не менее

$$
\frac{3}{4} 2^{-1} n^{-1} c_{6}^{-1} Q^{v_{0}-v_{1}} \mu I \gg Q^{v_{0}-v_{1}} \asymp Q^{n+1-(m(m+3) / 2) \gamma}
$$

многочленов, кластеры $M_{m+1}$ которых удовлетворяют $(6)$, причем никакие многочлены в этом наборе не будут совпадать. Положим $k=m+1$. Теорема 1 доказана. 


\section{СПИСОК ЦИТИРОВАННОЙ ЛИТЕРАТУРЫ}

[1] A. Baker, W. M. Schmidt, Proc. London Math. Soc. (3), 21 (1970), 1-11. [2] В. Г. Спринджук, Проблема Малера в метрической теории чисел, Наука и техника, Минск, 1967. [3] K. Mahler, Michigan Math. J., 11:3 (1964), 257-262. [4] V. V. Beresnevich, V. I. Bernik, F. Götze, Compositio Math., 146:5 (2010), 1165-1179. [5] Y. Bugeaud, M. Mignotte, Proc. Edinb. Math. Soc. (2), 47:2 (2004), 553-556. [6] Y. Bugeaud, M. Mignotte, Int. J. Number Theory, 6:3 (2010), 587-602. [7] Y. Bugeaud, A. Dujella, Bull. Lond. Math. Soc., 43:6 (2011), 1239-1244. [8] Y. Bugeaud, A. Dujella, Root Separation for Reducible Integer Polynomials, arXiv: math.NT/1306.2128v1. [9] В.В. Бересневич, В. И. Берник, Ф. Гетце, Докл. НАН Беларуси, 54:5 (2010), 21-23.

\section{Н. В. Бударина}

Хабаровское отделение Института прикладной математики ДВО РАН

E-mail: buda77@mail.ru

\section{Ф. Гетце}

Bielefeld University, Германия

E-mail: goetze@math.uni-bielefeld.de
Поступило

03.07.2013 